\title{
A microfluidic device for array patterning by perpendicular electrokinetic focusing
}

\author{
Dietrich Kohlheyer · Sandeep Unnikrishnan • \\ Geert A. J. Besselink · Stefan Schlautmann · \\ Richard B. M. Schasfoort
}

Received: 15 May 2007 / Accepted: 30 July 2007 / Published online: 22 August 2007

(C) Springer-Verlag 2007

\begin{abstract}
This paper describes a microfluidic chip in which two perpendicular laminar-flow streams can be operated to sequentially address the surface of a flowchamber with semi-parallel sample streams. The sample streams can be controlled in position and width by the method of electrokinetic focusing. For this purpose, each of the two streams is sandwiched by two parallel sheath flow streams containing just a buffer solution. The streams are being electroosmotically pumped, allowing a simple chip design and a setup with no moving parts. Positioning of the streams was adjusted in real-time by controlling the applied voltages according to an analytical model. The perpendicular focusing gives rise to overlapping regions, which, by combinatorial (bio) chemistry, might be used for fabrication of spot arrays of immobilized proteins and other biomolecules. Since the patterning procedure is done in a closed, liquid filled flow-structure, array spots will never be exposed to air and are prevented from drying. With this device configuration, it was possible to visualize an array of 49 spots on a surface area of $1 \mathrm{~mm}^{2}$. This article describes the principle, fabrication, experimental results, analytical modeling and numerical simulations of the microfluidic chip.
\end{abstract}

Keywords Micro array $\cdot$ Laminar-flow $\cdot$ Microfluidic . Electrokinetic-focusing

D. Kohlheyer $(\bowtie)$. S. Unnikrishnan · G. A. J. Besselink ·

S. Schlautmann - R. B. M. Schasfoort

University of Twente, MESA+, P.O. Box 217,

7500AE Enschede, The Netherlands

e-mail: d.kohlheyer@utwente.nl

\section{Introduction}

The research area of lab-on-a-chip or micro-total-analysis systems has grown rapidly in the last years (Tudos et al. 2001; Vilkner et al. 2004). Miniaturization of (bio) chemical analysis and synthesis systems has important advantages such as the small amount of reagents needed, improved reproducibility and speed of analysis (Huikko et al. 2003; Sato et al. 2003; Erickson and Li 2004). As microfluidics operate at low Reynolds numbers $(R e<$ 2,000) (Reynolds 1883) the position and the width of a laminar-flow stream can be varied by sandwiching the stream in-between two parallel flowing sheath flow streams. This technique, in case of pressure driven systems, is known as hydrodynamic focusing (Knight et al. 1998). Hydrodynamic focusing has been applied for particle sorting, alignment and counting (Dittrich and Schwille 2003), with multiple focusing as fluidic switches (Lee et al. 2001) but also for a confined patterning of metal and polyelectrolyte structures inside microfluidic channels (Kenis et al. 1999, 2000; Shchukin et al. 2004). Hydrodynamic focusing is an interesting method to pattern ligand arrays onto biosensors as compared with alternative microfluidic techniques such as stamping and spotting (Chiu et al. 1999; Bernard et al. 2001; Juncker et al. 2001). It is possible to guide cell and protein containing streams inside a flow-chamber and to immobilize the ligands in parallel lanes (Regenberg et al. 2004). Furthermore, by removing a microfluidic PDMS structure from the substrate and applying it a second time turned by $180^{\circ}$, more complex two-dimensional arrays can be formed (Takayma et al. 1999; Brevig et al. 2003). A recent overview about different patterning methods by means of microfluidics and was published by Situma et al. (2006). Dusseiller et al. (2005) used hydrodynamic focusing to position proteins 
and vesicles in two directions. An alternative to hydrodynamic focusing is electrokinetic focusing in which electroosmotic-flow (EOF) is used as the fluid pumping mechanism instead of pressure driven flow. EOF is the motion of the bulk liquid in response to an applied electrical field in a capillary, which is extensively used in capillary-electrophoresis systems (Effenhause et al. 1997). The usage of EOF implies several advantages compared with pressure driven systems: no valves or other moving parts are involved, which allows a simple chip structure and a fast and accurate switching between different flowrates. However, EOF is mainly usable in combination with aqueous solutions and other polar solvents, and in most cases voltages up to several $100 \mathrm{~V}$ are required to generate adequate flow rates. Furthermore, electrochemical phenomena such as electrolysis and unwanted bubble formation may disturb flow behavior. To remove gas produced during the electrolysis the electrodes have to be placed outside the actual flow-structure in the open fluid reservoirs. It has been successfully demonstrated to vary three and five parallel fluid streams in position and width inside in laminar-flow chamber by electrokinetic focusing (Besselink et al. 2004; Kohlheyer et al. 2005).

Described here is a sealed microfluidic structure, which can be used to pattern two-dimensional biochemical arrays. It is not necessary to remove the flow-structure and therefore, array spots are never exposed to air and are prevented from drying. Due to the mentioned advantages electrokinetic focusing is used to position two perpendicular sample containing streams inside a laminar-flow chamber.

As illustrated in Fig. 1, each chip consists of a microfluidic network of six inlet channels, a wider cross-shaped flow-chamber and two outlet channels. The position and the width of two sample streams (Fig. 1, sample 1 and sample 2) can be varied inside the laminar-flow chamber. This is achieved by adjusting the flux ratio of the sheath flow streams sandwiching each sample stream. These sheath flow streams contain only buffer solution. The two sample streams can be operated to sequentially (1a and $1 \mathrm{~b}$ ) coat the chip surface. Since EOF is used for pumping the liquids the required electrical voltages have to be applied outside the actual flow structure by electrodes inside the fluid reservoirs. During operation three voltages are calculated and applied to the electrodes in the active flow direction in real-time, while the electrodes in the perpendicular direction are set to floating (i.e., act as disconnected). To move the sample stream inside the flow chamber, the voltages applied to the sheath flow streams are controlled. Increasing one voltage and decreasing the other voltage, increases the volume flow of the first one but decreases the flow of the second sheath. This results in unequal widths of the sheath flow streams inside the chamber, thus changing the position of the sandwiched sample stream. Here the principle, fabrication and the experimental characterization of the device are described and discussed. The chip was used to verify the principle of forming a micro array by applying electrokinetic focusing of two perpendicular streams, each forming lanes on the chip surface resulting in a 2D grid. To control the required voltage settings an analytical model was applied.

\section{Experimental}

\subsection{Micro fabrication}

The fabrication is described briefly since only standard cleanroom fabrication techniques were applied. Most of the cleaning and intermediate steps are not mentioned. The microfluidic chip consists of two bonded plates. The silicon top plate contains the microfluidic channels as well as inlet and outlet holes while the bottom Pyrex glass plate is left unprocessed.

To fabricate the $10 \mu \mathrm{m}$ deep channel structure, a standard photolithography step was carried out on a $100 \mathrm{~mm}$ diameter $<100>$ silicon wafer. The developed photoresist served as an etching mask during the following deepreactive-ion-etch (DRIE) step of silicon. After removing the photoresist the silicon wafer was coated with $250 \mathrm{~nm}$ low-stress silicon-nitride $(\mathrm{Si}-\mathrm{N})$ layer during a low-pressure chemical vapor deposition. This $\mathrm{Si}-\mathrm{N}$ layer was structured after a second photolithography step on the backside of the wafer by plasma etching, to form a mask layer for the inlet and outlet holes. These through-holes were etched in a $2 \% \mathrm{KOH}$ bath at $75^{\circ} \mathrm{C}$ while the $\mathrm{Si}-\mathrm{N}$ layer protected the rest of the wafer. The $\mathrm{Si}-\mathrm{N}$ layer was

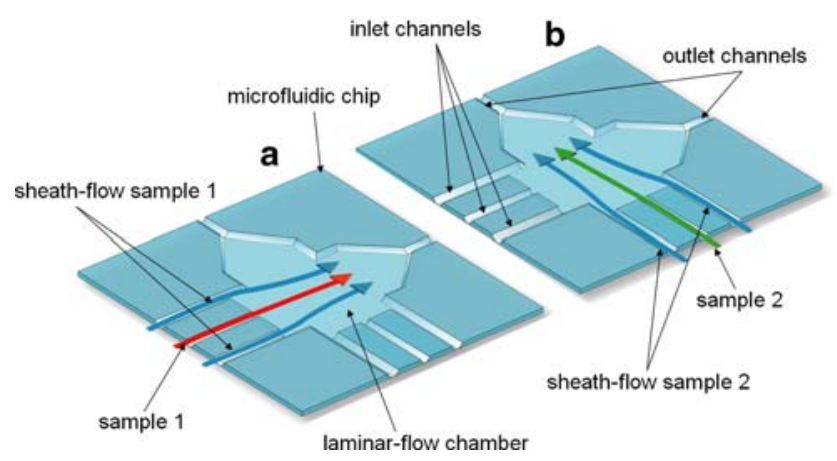

Fig. 1 Illustration of the principle layout of the chip. Two laminar flow streams are focused by two parallel sheath flows. These sample streams can operate in terms to coat the chip surface. First, lanes are generated in one direction by steering the sample to different positions (a). Second, perpendicular lanes are generated (b). The overlapping regions form the array. Fluid flow is achieved by electroosmotic flow 
removed by etching in 50\% hydrofluoric acid (HF). Since silicon is a semi-conducting material it has to be completely covered with an isolating layer when applying electroosmotic flow with high voltages involved. The isolation layer was achieved by a wet oxidation step at $1,150^{\circ} \mathrm{C}$ to grow $1 \mu \mathrm{m}$ layer of $\mathrm{SiO}_{2}$. The processed oxidized silicon wafer was bonded to a Pyrex glass wafer by anodic bonding and finally diced into single chips.

Figure 2 illustrates the three main fabrication steps involved in the process (flow-structure etching, inlet/outlet etching and bonding). The chip size is $15 \times 15 \mathrm{~mm}^{2}$ and the thickness is $1 \mathrm{~mm}$. The surface area for patterning, e.g., bio-arrays is about $1 \mathrm{~mm}^{2}$ and the channel widths differ from 150 to $30 \mu \mathrm{m}$ and the channel height is $10 \mu \mathrm{m}$. The fabricated microfluidic chip is shown in Fig. 3 a and the flow-chamber in the close-up Fig. 3b.

\subsection{Materials}

Bovine serum albumin (BSA), fluorescein isothiocyanate and Hepes were obtained from Sigma (Sigma-Aldrich Chemie BV, Zwijndrecht, The Netherlands). Rhodamine B labeled dextrane (MW 10,000) was obtained from Molecular Probes (Invitrogen BV, Breda, The Netherlands). All other chemicals were purchased from VWR International (Darmstadt, Germany) and were of analytical grade. BSA (30 $\mathrm{mg} \mathrm{ml}^{-1}$ in $0.5 \mathrm{M}$ sodium borate, $\mathrm{pH}$ 9.0) was fluorescently labeled by treatment for $30 \mathrm{~min}$ at room temperature with (a 60-fold molar excess of) fluorescein isothiocyanate, followed by gel filtration over a PD-10 column (Amersham Biosciences, Uppsala, Sweden). A solution of $10 \mathrm{mM}$ Hepes in demineralized water was prepared and adjusted to $\mathrm{pH} 8.0$.

\subsection{Setup and methods}

For experimental work, the flow structure was flushed several minutes with $1 \mathrm{M} \mathrm{NaOH}$ and afterwards with demineralized water to clean the channels and to ensure reproducible EOF conditions. The outside of the chip was

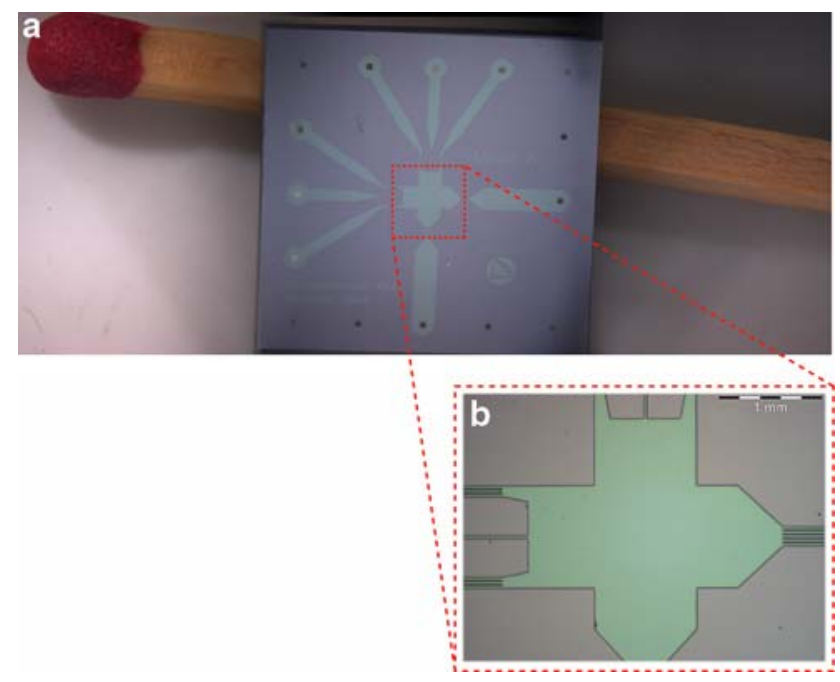

Fig. 3 Photographs of the fabricated chip: the chip is $15 \times 15 \mathrm{~mm}^{2}$ in size with a thickness of $1 \mathrm{~mm}$ (a). The actual flow chamber, which is used for array patterning has a surface area of $1 \mathrm{~mm}^{2}(\mathbf{b})$

cleaned with isopropanol and dried with nitrogen. The fabricated chip was mounted into an in-house fabricated holder with integrated fluid reservoirs and platinum electrodes.

The holder (Fig. 4) consists of two parts, which fixate the chip in-between while the glass side of the chip faces downwards to the square opening. Sealing between the chip and the holder is achieved with a laser-cut rubber plate of $1 \mathrm{~mm}$ thickness. All holder reservoirs were filled with $10 \mathrm{mM}$ Hepes ( $\mathrm{pH}$ 8.0) and a conventional syringe was used to apply vacuum to several reservoirs to remove air bubbles and fill the microfluidic network with the buffer solution. All reservoirs were emptied and refilled with buffer to ensure equal fluid heights. For stream visualization the sample stream reservoirs were filled with $1 \mathrm{mg} \mathrm{m}^{-1}$ Rhodamine $B$ in $10 \mathrm{mM}$ Hepes buffer and $1 \mathrm{mg} \mathrm{m}^{-1}$ Fluorescein labeled BSA in Hepes buffer, respectively. The integrated electrodes were connected to two computercontrolled power supplies (IBIS 411, microfluidic control unit; IBIS Technologies B.V., Hengelo, The Netherlands) supplying eight independent voltage channels (up to $1 \mathrm{kV}$ ). This holder was placed on an inverted microscope
Fig. 2 The fabrication process can be divided into three steps: (1) etching the channel network into a silicon wafer. (2) Etching the channel inlets from the wafer backside. (3) Bonding a Pyrex glass wafer to close the structure

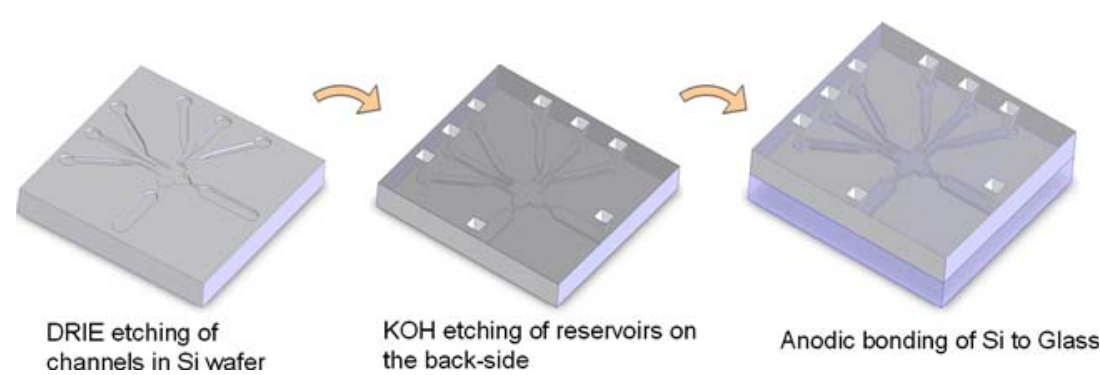


(Olympus IX51, Olympus Nederland B.V. The Netherlands) equipped with a mercury lamp and appropriate fluorescent filter cubes for visualization of the fluid streams. A digital color camera (ColorView II, Soft Imaging Systems, Münster, Germany) was attached to the microscope and images were captured during experimental work with the imaging software package Analysis (analySIS 5, Soft Imaging Systems, Münster, Germany).

The positions of the two samples streams were varied by controlling the voltage settings with a in-house written Labview program (LabVIEW 7 Express, National Instruments). Therefore, an analytical model, which is an analogue of EOF to an electrical network, was developed and embedded into the software allowing real-time control of the sample stream positions and widths by calculating the corresponding voltage settings.

The distribution of the electrical field inside the microfluidic chip and EOF operation was simulated with the CFD-ACE+ software (CFD Research Corporation, Huntsville, USA). The microfluidic device was approximated as a 2D structure with approximately 7,000 grid-cells. The simulation was performed with "no-slip conditions at walls". The electrical boundary conditions were obtained from the analytical model. Residual convergence criteria were set to $10^{-4}$. The electroosmotic mobility was set to $5 \times 10^{-8} \mathrm{~m}^{2} /(\mathrm{V} \mathrm{s})$ for guiding as well as the sample stream (Lion et al. 2003).

\subsection{Analytical model}

An analytical model was developed to calculate the required voltage settings for any sample stream position, velocity and width. This model is based on earlier presented results (Qiao and Aluru 2002; Ajdari 2003; Besselink et al. 2004; Kohlheyer et al. 2005). Therefore, it is described only briefly. To simplify the calculations, the cross-shaped flow chamber was neglected and a straight channel was assumed. Furthermore entrance effects were neglected. Figure 5a shows the relevant geometrical parameters, which were used for the calculations. An analogy of electroosmotic flow to an electrical network was developed as illustrated in Fig. 5b.

Due to mass conservation, the fluid stream through each inlet channel is proportional to its corresponding fluid stream inside the flow chamber. Therefore, the position $x_{2}$ and the width $w_{2 \mathrm{ch}}$ of the sample stream inside the chamber can be expressed by:

$X_{2}=\frac{\phi_{1}+\frac{1}{2} \phi_{2}}{\phi_{1}+\phi_{2}+\phi_{3}} \boldsymbol{w}_{\mathrm{ch}}$

and

$\boldsymbol{w}_{2 \mathrm{ch}}=\frac{\phi_{2}}{\phi_{1}+\phi_{2}+\phi_{3}} \boldsymbol{w}_{\mathrm{ch}}$
Fig. 4 An in-house fabricated holder was used to mount the chip on an inverted microscope with fluorescent equipment. This holder has integrated platinum electrodes for electrical connections. Sealing between the chip and the lid was achieved by a laser cut rubber plate

Fig. 5 This figure is showing the technical layout with relevant geometrical and flow parameters (a). To calculate the voltages required for the electroosmotic flow an electrical network was used. Each channel is represented by an electrical resistance
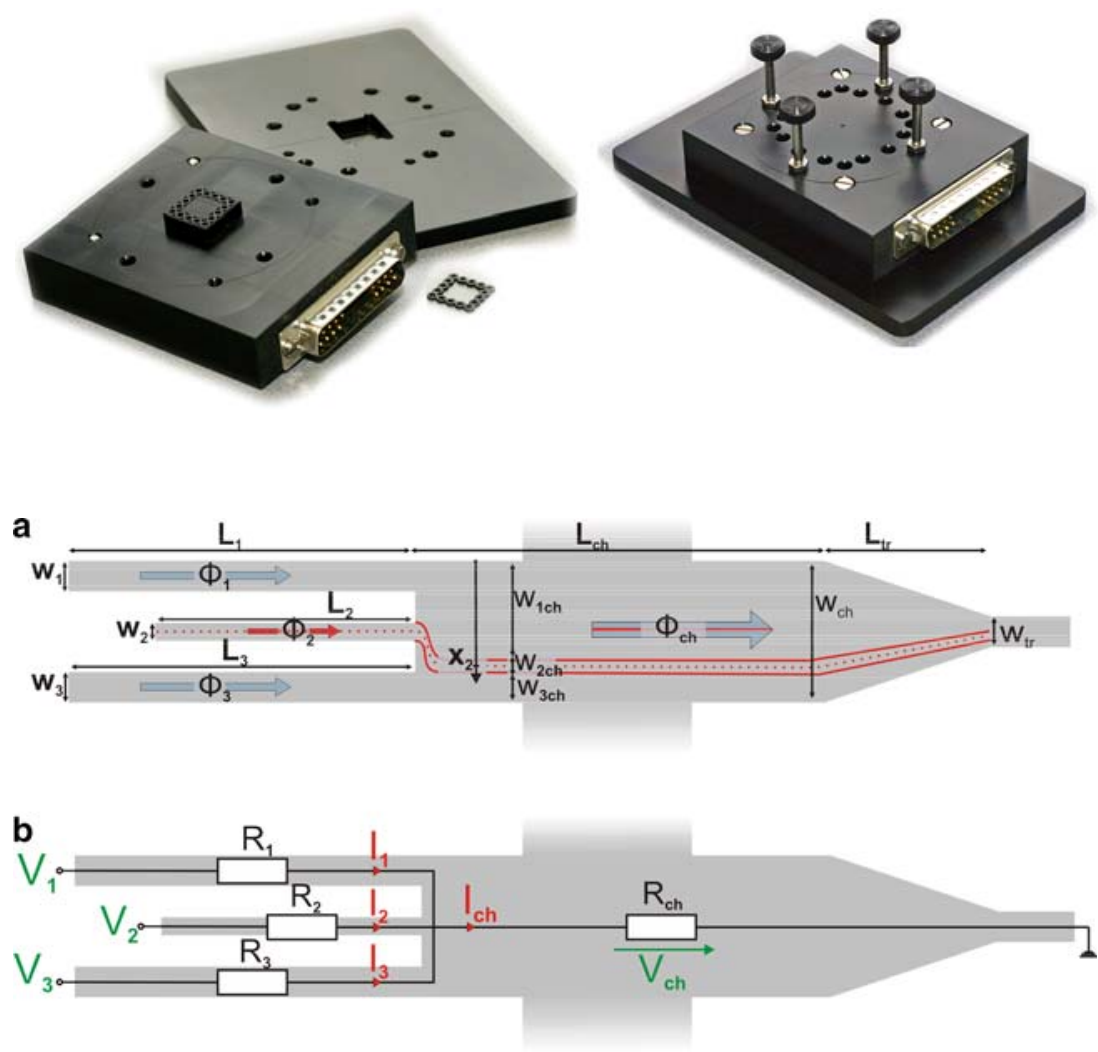
Table 1 Voltages used during experimental work

\begin{tabular}{llll}
\hline Position & $V_{1} / V$ (sheath 1$)$ & $V_{2} / V$ (sample) & $V_{3} / V$ (sheath 2$)$ \\
\hline 1 & 118 & 133 & 241 \\
2 & 133 & 133 & 226 \\
3 & 149 & 133 & 210 \\
4 & 164 & 133 & 195 \\
5 (center) & 180 & 133 & 180 \\
6 & 195 & 133 & 164 \\
7 & 210 & 133 & 149 \\
8 & 226 & 133 & 133 \\
9 & 241 & 133 & 118 \\
\hline
\end{tabular}

The velocity was constant at $1 \mathrm{~mm} \mathrm{~s}^{-1}$ for all settings

where $w_{\mathrm{ch}}$ is the width of the flow chamber and $\Phi_{i}$ the flow rate through an inlet channel.

The flow rate $\Phi_{i}$ through each channel is given by:

$\phi_{i}=\mu_{\mathrm{EOF} i} A_{i} \frac{\boldsymbol{V}_{i}-\boldsymbol{V}_{\mathrm{ch}}}{\boldsymbol{L}_{i}}$

where $\mu_{\mathrm{EOF}}$ is the electroosmotic mobility, $A_{\mathrm{i}}$ the crosssectional area and $V_{\mathrm{i}}$ the voltage applied to each inlet reservoir and $L_{\mathrm{i}}$ the channel length. $V_{\mathrm{ch}}$, the voltage drop across the chamber, can be calculated using:

$\boldsymbol{V}_{\mathrm{ch}}=\frac{\frac{V_{1}}{\boldsymbol{R}_{1}}+\frac{V_{2}}{\boldsymbol{R}_{2}}+\frac{V_{3}}{\boldsymbol{R}_{3}}}{\frac{1}{\boldsymbol{R}_{c h}}+\frac{1}{\boldsymbol{R}_{1}}+\frac{1}{\boldsymbol{R}_{2}}+\frac{1}{\boldsymbol{R}_{3}}}$.

Knowing the electrical properties such as conductivity and the geometrical parameters, the electrical resistance of the channels $R_{i}$ and the electrical resistance of the chamber $R_{\mathrm{ch}}$ the mentioned equations can be solved. The analytical model is a useful tool for calculating the voltages that are needed for obtaining the desired sample stream position and width.

\section{Results and discussion}

\subsection{Experimental array visualization}

For obtaining an impression of the flow behavior and visualizing the array geometry, two fluorescent components were chosen as flow marker, namely Rhodamine B and fluorescein labeled BSA. After prefilling the chip, Rhodamine B was added to the first sample reservoir. The
Fig. 6 These images were achieved by superimposing several photographs taken during the experimental work. In order to obtain this, a $1 \mathrm{mg} \mathrm{ml}^{-1}$ Rhodamine B containing sample stream was steered to nine different parallel positions (a). Each lane was recorded separately (illustrated by the small images above figure a and right to $\mathbf{b}$ ). In a second step a sample containing $1 \mathrm{mg} \mathrm{ml}^{-1}$ Fluorescein labeled BSA was positioned in the perpendicular direction (b). All images were merged to visualize the generated array (c). The overlapping regions form the actual regions of interest (ROI)
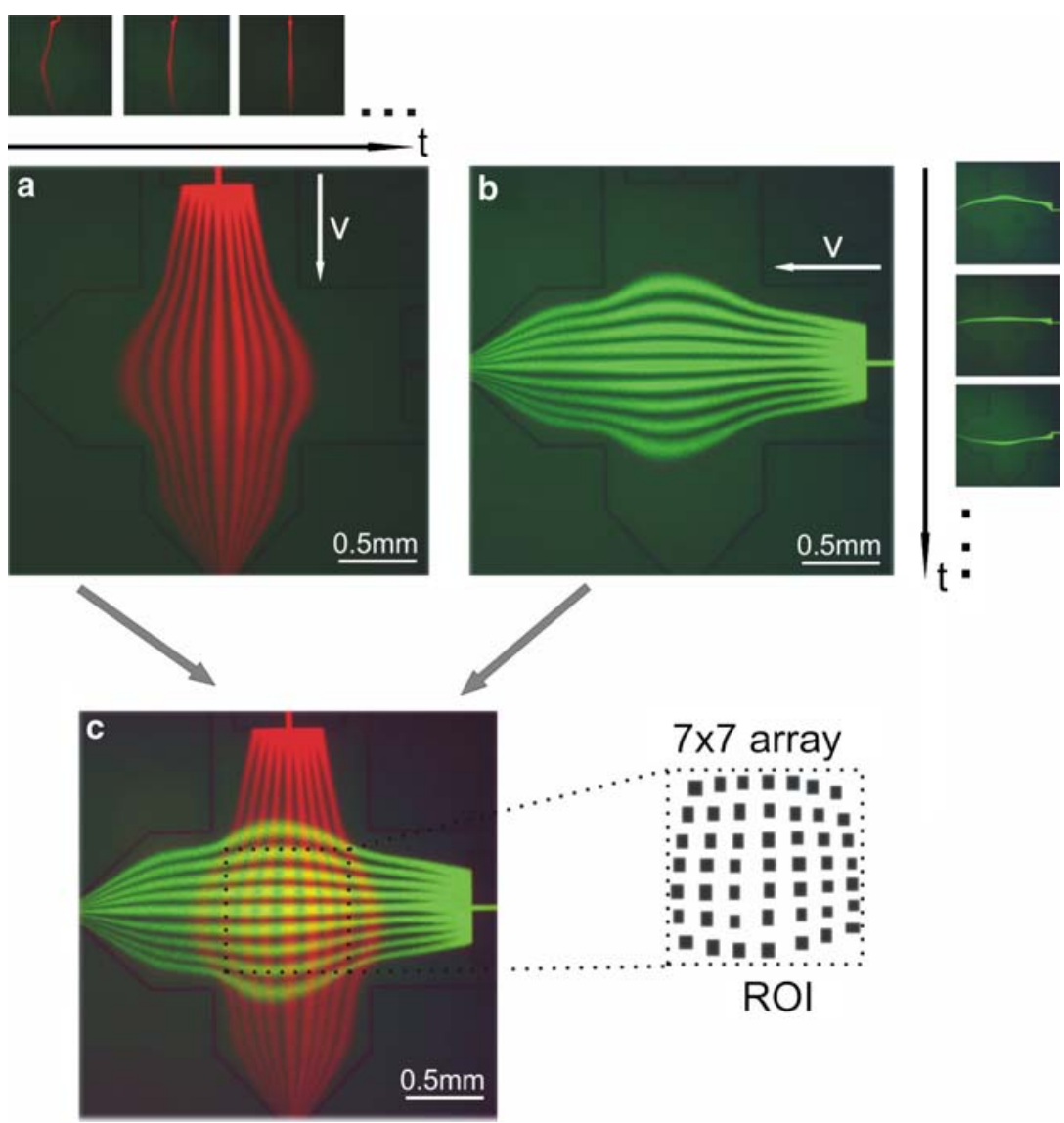
sample stream thickness was focused to approximately $50 \mu \mathrm{m}$. Due to diverging flow lines inside the cross shaped flow chamber, the sample stream tend to broaden near the sides. Therefore, the thickness of $50 \mu \mathrm{m}$ was only valid along the centerline of the chip. The flow velocity was set to $1 \mathrm{~mm} \mathrm{~s}^{-1}$. For this setting $133 \mathrm{v}$ was applied to the sample electrode and $180 \mathrm{v}$ to the sheath flow electrodes (valid for center position). When moving the sample inside the flow chamber, the voltages applied to the sheath flow electrodes were adjusted, keeping the voltage ratio constant to prevent altering the total current and hence the velocity (Table 1).

As shown in Fig. 6a, the Rhodamine B containing sample was sequentially positioned to nine different lanes forming the first dimension of the array. However, it has to be pointed out that no immobilization of Rhodamine B molecules was performed. Several photographs were taken during the experimental run and were merged to an image overlay for visualization of the sample streams. The voltage configuration was altered in the next patterning step. For this purpose, the electrical connections were changed from that of the first sample to that of the second sample and guiding channels. Now the sample containing BSA was guided to nine positions resulting in a second set of parallel lanes as shown in Fig. 6b. All 18 images were superimposed to one image to visualize the possible array geometry. In Fig. 6c it can be seen, that due to the curvature flow of the sample stream the array spots are not uniformly sized and positioned. However, if the highly broadened outer streams are excluded, an acceptable micro array of 49 spots was generated. This could be a useful format for bio-assay applications. Using image-based software, regions of interest (ROIs) could be placed inside the generated array spots. The cross-shaped flow chamber is not ideal, since the curvature flow caused by non-parallel electrical current streamlines is broadening the flow. However, the usage of a closed system instead of earlier presented results with removable PDMS cover layers will be advantageous for using proteins and other more fragile components as drying induced denaturation of biomolecules can be prevented.

Several principles based on combinatorial (bio) chemistry have been described in the literature, which could be a relevant application for this technique. DNA-directed immobilization (DDI), for example, employs proteins tagged with specific single strands of DNA, to be hybridized to complementary DNA strands previously spotted on a solid substrate. DDI is a self-assembly method that is chemically mild, and leads to relatively high protein immobilization yields and homogeneous results (Wacker et al. 2004). The use of DDI has been described for the preparation of protein microarrays that were employed in chip-based multi analyte detection (Wacker and Niemeyer
2004). The device described in the present report might be useful in such an approach: First by applying streams of different oligonucleotides in one direction resulting in immobilization of these oligonucleotides in distinct lanes; Next by applying streams of the DNA-tagged proteins in the perpendicular direction enabling hybridization between complementary DNA strands and the connected immobilization of the DNA-tagged proteins thereby resulting in protein microarrays. To generate a DDI based microarray, as described above, the future chip device has to be supplemented with several inlet reservoirs to enable feeding with different immobilants. The usage of electroosmotic flow allows an easy switching between the separate inlet reservoirs to immobilize different components, such as oligonucleotides. The device described here has only two separate sample reservoirs to apply an orthogonal pattern in order to demonstrate the basic functions.

When switching between various positions, crosstalk of different components might occur between adjacent spots immobilized on the surface. This will be avoided by an

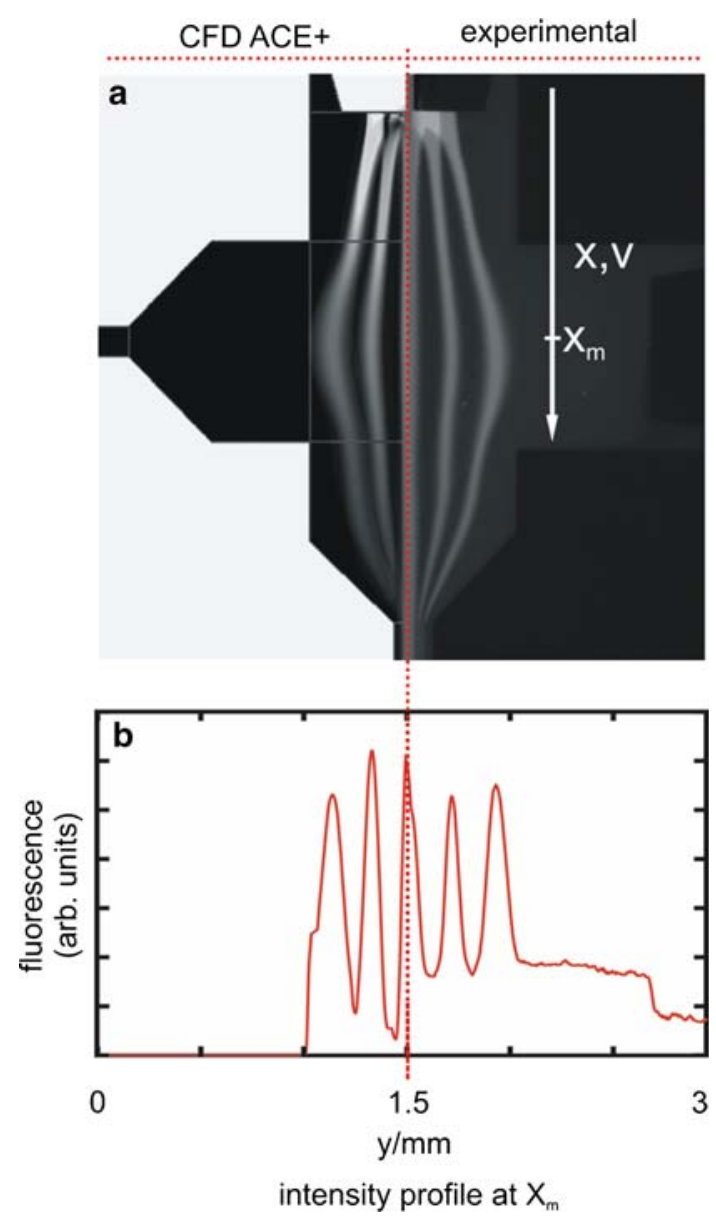

Fig. 7 Experimental results (a, right half) were compared with simulations (a, left half). For better comparison, an intensity profile measured at $X_{\mathrm{m}}$ is shown (b) 
Fig. 8 Illustration comparing simulation with experimental results: the cross shaped flow chamber has a distribution of the electrical field with nonparallel curved field lines. Electroosmotic flow is perpendicular to the flow resulting in a curved flow stream when applying unequal voltages $V_{1}$ and $V_{3}$

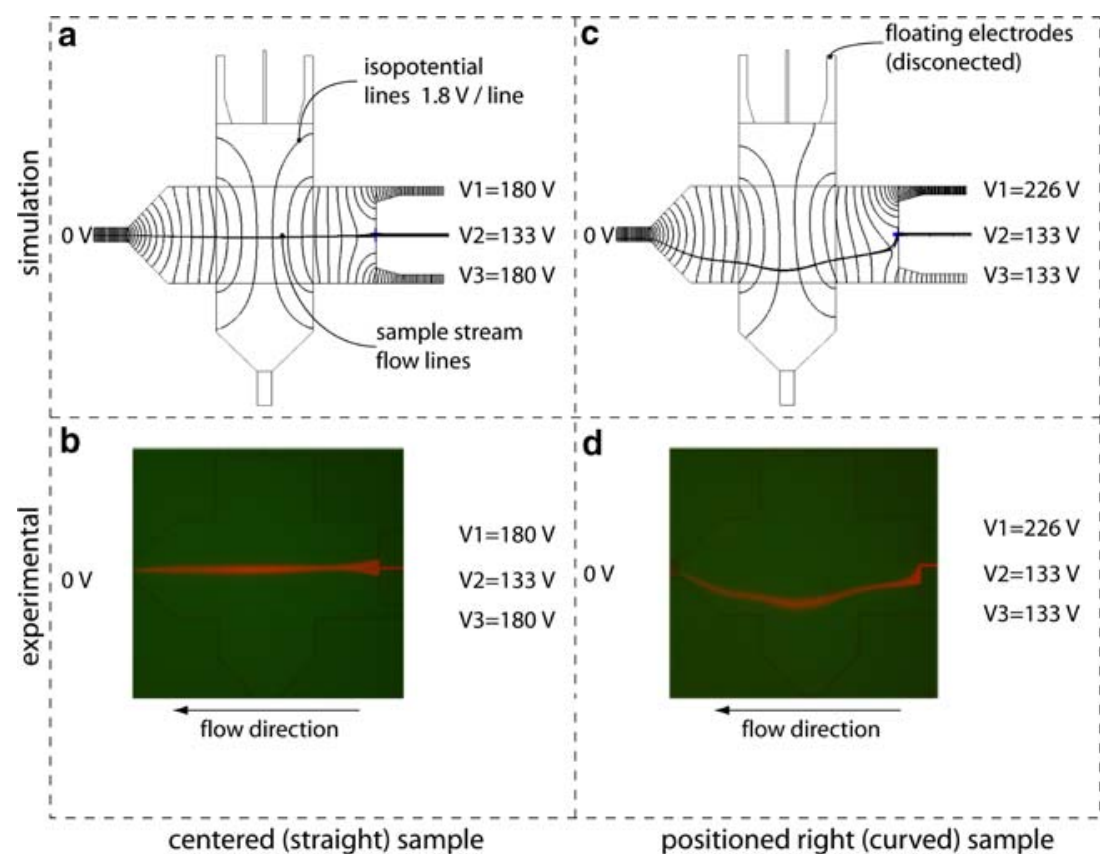

intermediate step, where an inlet reservoir with clean buffer solution is activated before moving the sample stream towards its next position. Crosstalk can also be caused by sample stream broadening due to diffusion, which can lead to overlapping between neighboring lanes. The extent of stream broading depends on flow-rate and the diffusion constant of the applied component and can be prevented by choosing proper lane pitches.

\subsection{Simulations}

To verify the experimental results as well as the analytical model, the system was simulated using a 2D model of the flow structure. Figure 6 shows a comparison between the simulation and the experimental result (left half: simulation, right half: experimental). Also shown is an intensity line profile from the image. These results indicate a good agreement between simulation and experimental results and, furthermore, prove the usefulness of the model in predicting stream positions. The higher background level in the right part of the intensity plot is due to a auto fluorescence caused by the silicon oxide layer inside the chip (Fig. 7).

The curvedness and broadening of the sample stream increases to the sides is a result of the inhomogeneous distribution of the electrical field. As can be derived from Fig. 8a, b since EOF stream lines are perpendicular to the electrical field lines, the flow line of a centered sample will remain straight. However, when unequal voltages $V_{1}$ and $V_{3}$ are applied $\left(V_{1}+V_{3}=\right.$ constant $)$ the sample stream is guided to the lower side (Fig. 8c, d), leading to a curved sample stream. Furthermore the lower field strength and lower velocity near the sides of the flow chamber result in a broadening of the sample stream.

\section{Conclusions}

An EOF driven chip system that can be used for patterning a surface in an array-like manner, was demonstrated. To visualize the possible array dimensions, two fluorescent flow markers were used and images were superimposed. Two perpendicular laminar flow streams could be sequentially operated to address the chip surface with parallel lanes in a perpendicular direction which results in two overlapping grids, thus generating an array of 49 spots or more on a surface area of $1 \mathrm{~mm}^{2}$. No immobilization was performed yet; however, the technology is promising. Since electroosmotic flow is a pumping mechanism where no pumps and moving parts are involved, the setup is easy and straight forward to handle. An analytical model proved to be useful in predicting the stream positions and widths by calculating the required voltages. This technology is a new line spotting tool for generating, e.g., biochemical micro arrays without exposure of fragile biomolecules to air.

Acknowledgments We acknowledge the funding of this research by the technology foundation STW as part of the "Proteomics on a chip for monitoring autoimmune diseases" project. Furthermore, special thanks to Hans de Boer for the technical support and fabrication of the chip holder. 


\section{References}

Ajdari A (2003) Steady flows in networks of microfluidic channels: building on the analogy with electrical circuits. C R Physique 5:539-546

Bernard A, Michel B et al (2001) Micromosaic immonuassays. Anal Chem 73:8-12

Besselink GAJ, Vulto P et al (2004) Electroosmotic guiding of sample flows in a laminar flow-chamber. Electrophoresis 25:3705-3711

Brevig T, Krühne U et al (2003) Hydrodynamic guiding for addressing subsets of immobilized cells and molecules in microfluidic systems. BMC Biotechnol 3(10)

Chiu DT, Jeon NL et al (1999) Patterned deposition of cells and proteins onto surfaces by using three-dimensional microfluidic systems. Proc Natl Acad Sci USA 97(6):2408-2413

Dittrich PS, Schwille P (2003) An integrated microfluidic system for reaction, high-sensitivity detection, and sorting of fluorescent cells and particles. Anal Chem 75:5767-5774

Dusseiller MR, Niederberger B et al (2005) A novel crossed microfluidic device for the precise positioning of proteins and vesicles. Lab Chip 5:1387-1392

Effenhause CS, Bruin GJ et al (1997) Integrated chip-based capillary electrophoresis. Electrophoresis 18:2203-2213

Erickson D, Li D (2004) Integrated microfluidic devices. Anal Chim Acta 507(1):11

Huikko K, Kostiainen R et al (2003) Introduction to micro-analytical systems: bioanalytical and pharmaceutical applications. Eur J Pharm Sci 20(2): 149

Juncker D, Schmid H et al (2001) Soft and rigid two-level microfluidic networks for patterning surfaces. J Micromech Microeng 11:532-541

Kenis PJA, Ismagliov RF et al (1999) Microfabrication inside capillaries using multiphase laminar flow patterning". Science 285:83-85

Kenis PJA, Ismagilov RF et al (2000) Fabrication inside microchannels using fluid flow. Acc Chem Res 33(12):841-847

Knight JB, Vishwanath A et al (1998) Hydrodynamic focusing on a silicon chip: mixing nanoliters in microseconds. Phys Rev Lett 80(17):3863-3866
Kohlheyer D, Besselink GAJ et al (2005) Electroosmotically controllable multi-flow microreactor. Microfluid Nanofluid 1(3):242-248

Lee GB, Hwei BH et al (2001) Micromachined pre-focused $\mathrm{M} \times \mathrm{N}$ flow switches for continuous multi-sample injection. J Micromech Microeng 11:654-661

Lion N, Rohner TC et al (2003) Microfluidic systems in proteomics. Electrophoresis 24:3533-3562

Qiao R, Aluru NR (2002) A compact model for electroosmotic flows in microfluidic devices. J Micromech Microeng 12:625-635

Regenberg B, Krühne U et al (2004) Use of laminar flow patterning of miniaturized biochemical assays. Lab Chip 4:654-657

Reynolds O (1883) On the experimental investigation of the circumstances which determine whether the motion of water shall be direct or sinuous, and the law of resistance in parallel channels. Philos Trans R Soc 174:935-982

Sato K, Hibara A et al (2003) Microchip-based chemical and biochemical analysis systems. Adv Drug Deliv Rev 55(3):379

Shchukin DG, Kommireddy DS et al (2004) Polyelectrolyte micropatterning using a laminar flow microfluidic device. Adv Mater 16(5):389-393

Situma C, Hashimoto M et al (2006) Merging microfluidics with microarray-based bioassays. Biomol Eng 23:213-231

Takayma S, McDonald JC et al (1999) Patterning cells and their enviroment using multiple laminar fluid flows in cappillary networks. Proc Natl Acad Sci USA 96:5545-5548

Tudos AJ, Besselink GAJ et al (2001) Trends in miniaturized total analysis systems for point-of-care testing in clinical chemistry. Lab Chip 1:83-95

Vilkner T, Janasek D et al (2004) Micro total analysis systems. recent developments. Anal Chem 76:3373-3386

Wacker R, Niemeyer CM (2004) DDI- $\mu$ FIA-A readily configurable microarray-fluorescence immunoassay based on DNA-directed immobilization of proteins. ChemBioChem 5:453-459

Wacker R, Schröder H et al (2004) Performance of antibody microarrays fabricated by either DNA-directed immobilization, direct spotting, or streptavidin-biotin attachment: a comparative study. Biochemistry 330:281-287 